\title{
Dynamic Voltage Restorer (DVR) Utilized for Enhancing the Voltage Quality in Medium Voltage Networks using Hybrid Power Source Setup
}

\author{
Ashish Tiwari ${ }^{1}$, Vikash Mohan ${ }^{2}$ \\ ${ }^{1}$ M.E Student, Department of Electrical \& Electronics Engineering, IES College of Technology, Bhopal \\ ${ }^{2}$ Assistant Professor, Department of Electrical \& Electronics Engineering, IES College of Technology, Bhopal
}

\begin{abstract}
This thesis talks about the execution of a Dynamic Voltage Restorer (DVR) utilized for enhancing the voltage Quality in medium voltage networks using hybrid power source setup. The DVR compensator is investigated as a Voltage Controller. Demonstrating of DVR and his control plan are proposed. The acquired results illustrate the exhibitions and the advantages of Dynamic Voltage Restorer used in Power system with auxiliary Distribution setup. This system will compensate voltage dips in medium voltage networks with secondary distribution configurations. The compensation will be done by using a dynamic voltage restorer (DVR) supplied through several series connected batteries. These batteries are charged by fuel cells. The electrical distribution network and the dynamic voltage restorer are modeled using Matlab / Simulink simulation environment in order to demonstrate the effectiveness of proposed equipment. The simulations carried out showed that the chosen configuration of DVR provides excellent voltage compensation capabilities. The simulator used for simulation of Dynamic Voltage Simulator is MATLAB SIMULINK. This proposal contains design, simulation also checking stability and other analysis of proposed model.
\end{abstract}

Keywords: Voltage Source Converter,point of common coupling,Active Power Filters,Battery Energy Storage Systems,Dynamic Voltage Restorer

\section{Introduction}

Of late, it could be watched that the advancement and ceaseless change of Electronic Components fabricating Determinate a complemented development rate of their utilization in both industrial and residential sector. Because of the certainty that these Receivers, in view of Electronic Component, are delicate to irritations of Voltage Waveform, even in the event that of a minor rebelliousness of Power Quality conveyed by the Power organizations, modern customers can endure gigantic budgetary misfortunes and the solace of private customers diminishes. Voltage Dips and Interruptions in power supply is one of the fundamental components that bring about these issues. A Voltage Dip is a passing decline in the Root Mean Square Voltage somewhere around 0,1 and 0,9 for every unit, with a term extending from a half cycle up to 1 minute[1,2]. It is created by Faults in Power System or by starting of expansive Induction Machines. It can hinders or glitch any Electronic Device which is a touchy burden. Pretty much as Flexible Ac Transmission Systems are utilized to enhance the unwavering quality and nature of Transmission Systems, this kind of Equipment taking into account Power Electronic Components can be utilized as a part of Power Distribution Systems with advantages for bringing answer for an extensive variety of issues. These Supplies, known as Custom Power Devices, can enhance the nature of power conveyed to clients. Dynamic Voltage Restorer (Dvr) is a Solid-State Power Controller able to give the vital Voltage Control at the load point of common coupling with The Electricity Distribution System for voltage quality change In the event that a DC Voltage source supplies the Dc Bus Of DVR, the compensator can infuse both Active and receptive power into the Power Dissemination Network keeping in mind the end goal to remunerate Server Voltage Plunges. This proposal examines the execution of the DVR for enhancing the Voltage Quality in medium Voltage Distribution Systems with auxiliary Distribution setup by upgrading Voltage Dip. Displaying and Control Scheme for DVR are depicted, including a point by point modeling of the Dynamic Voltage Restorer. Approval of Control Scheme and DVR model is helped out through simulations by utilizing SimPowerSystem, module of Matlab/Simulink.

\section{Dynamic Voltage Restorer}

The Dynamic Voltage Restorer is a Power electronic Converter based arrangement compensator that can secure touchy load from all Supply side unsettling influences with the exception of blackouts. This compensator utilizes IGBT strong state Power-electronic switches in a heartbeat width balanced inverter structure that infuses a set of three-stage air conditioning yield voltages in arrangement and synchronism with the Distribution line voltages. Since the voltages parameters sufficiency and stage point are variable hence the dynamic and receptive Power trade in the middle of DVR and system are controlled. An outside energy source or an energy stockpiling gadget of a legitimate limit is associated with de input terminal of the DVR keeping in mind the end goal to permit the dynamic Power trade in the middle of DVR and Distribution framework. By controlling the stage point and plenty fullness of infused voltages, the DVR can trade responsive Power with the Distribution framework without ac passive reactive components. [3, 4]

Basic setup of DVR:

The general setup of the DVR consists of: 


\section{International Journal of Science and Research (IJSR) \\ ISSN (Online): 2319-7064 \\ Index Copernicus Value (2015): 78.96 | Impact Factor (2015): 6.391}

1) Injection Transformers hat ensures galvanic isolation and simplify the protection equipment;

2) Line-filter that it used to reduce the switching harmonics generated by the converter;

3) Power converter that is a most likely a Voltage Source Converter with Pulse Width Modulation. The converter modulates the dc from the dc-link to ac voltages injected into the system;

4) DC-link and dc voltage source/energy storage device. The dc -link is used to synthesize an ac voltage into the Power line;

5) Mechanically and solid-state by-pass systems.

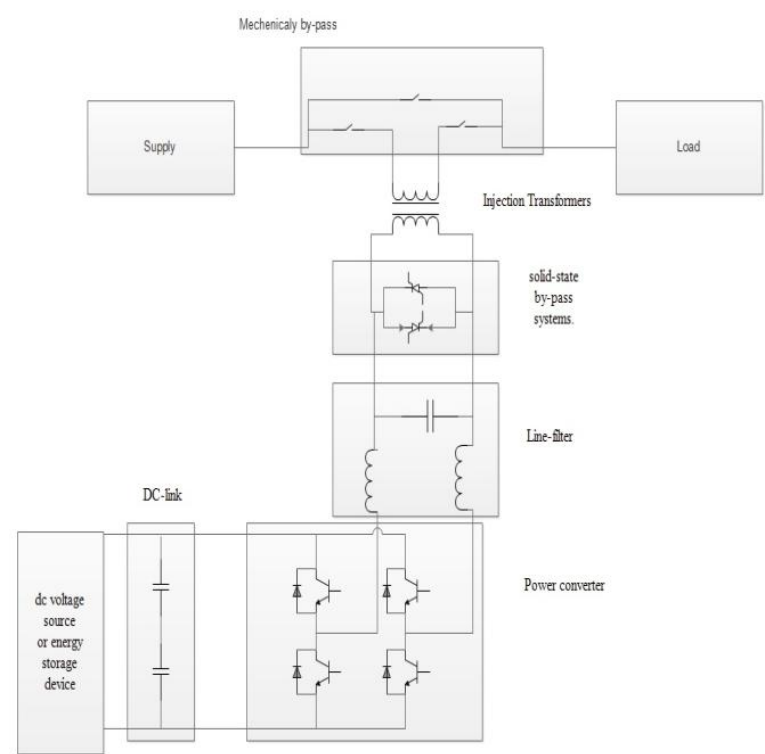

Figure 1: The basic elements of a DVR in a single-phase representation

\section{Equations related to DVR}

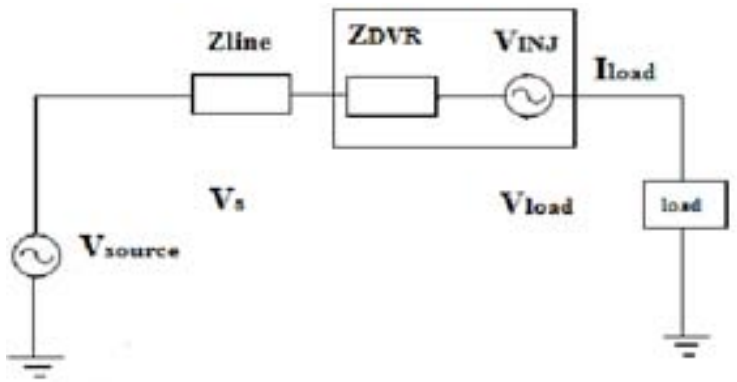

Figure 2: Equivalent circuit diagram of DVR

The System impedance Zth relies on upon the fault level of the Load bus. At the point when the System Voltage (Vth) drops, the DVR infuses an series Voltage VDVR through the Infusion Transformer so that necessary Load Voltage Magnitude $V_{L}$ can be kept up.

The series injected Voltage of the $V_{D V R}$ can be written as

$$
V_{D V R}=+Z_{T H} I_{L}-V_{T H}
$$

Where

$V_{L}$ : The desired Load Voltage Magnitude

$Z_{T H}$ : The Load impedance.
$I_{L}:$ The Load Current

$V_{T H}$ : The Network potential during fault condition

The load current $I_{L}$ is given by,

$$
I_{L}=\frac{\left[P_{L}+\mathrm{J} Q_{L}\right]}{\mathrm{V}}
$$

If the load voltage is considered as a reference, then equation (1) can be written in the following form:

$$
V_{D V R}(\cos \alpha+j \sin \alpha)=V_{L}(\cos 0+j \sin 0)+
$$

$$
Z_{t h} \cdot I_{L}(\cos (\beta-\theta)+j \sin (\beta-\theta))-V_{t h}(\cos \delta+j \sin \delta)
$$

Where: $\alpha, \beta$ and $\delta$ are the phase-angles of the $V_{D V R}, Z s h$ and Vth

Vectors and $\theta$ is the load Power factor, computed with:

$$
\theta=\tan ^{-1} \frac{Q_{L}}{P_{L}}
$$

The complex power injection of the DVR can be written as,

$$
S_{D V R}=V_{D V R} I_{L}
$$

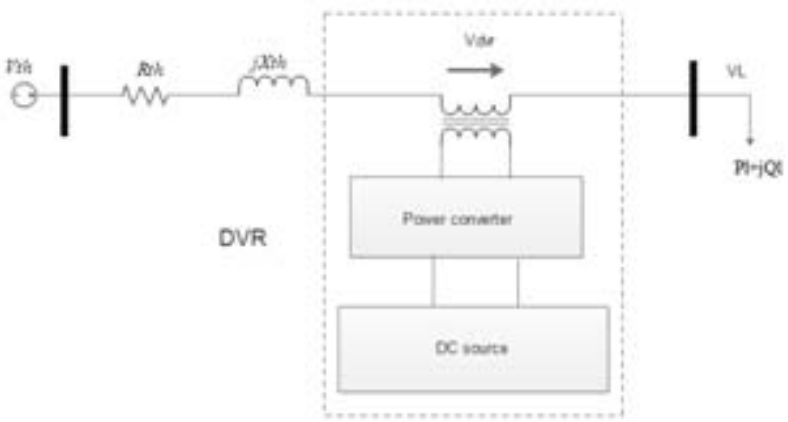

Figure 3: The DVR schematic connection diagram to the electricity Distribution network

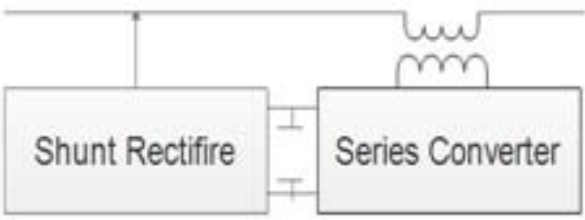

(a) Front connected shunt converter

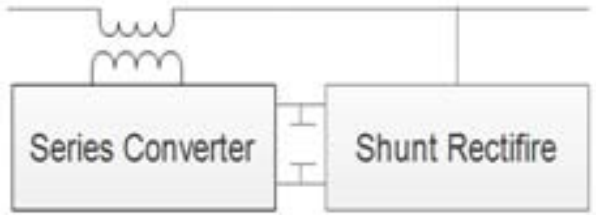

(b) Back connected shunt converter

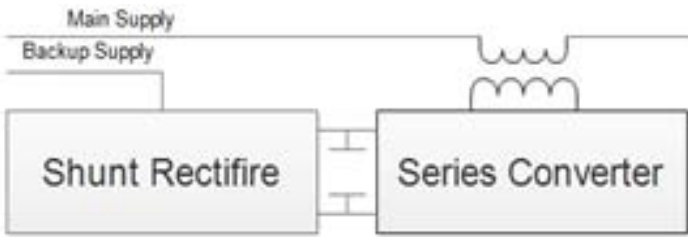

(c) Rectifier supplied through backup feeder

Figure 4: Types of connections to power source of the DVR: a) front connected shunt converter; b) back connected shunt converter; c) rectifier supplied through backup feeder

The dc voltage connection can be fueled by a energy 


\section{International Journal of Science and Research (IJSR) \\ ISSN (Online): 2319-7064 \\ Index Copernicus Value (2015): 78.96 | Impact Factor (2015): 6.391}

stockpiling framework or a rectifier supplied from the medium voltage system. Utilizing a rectifier, the dynamic voltage restorer can retain genuine Power from the system through the dc Bus thus will have the capacity to remunerate serious annoyances. The rectifier can be associated with the load side of the dynamic voltage restorer or to the Supply side [5, 9, and 10]. A third connection scheme can be achieved if the rectifier is supplied through another feeder. These three possible connections schemes are presented in figure 4. The single-phase equivalent circuit of the DVR with a LC filter, placed of the converter side of the Transformer to prevent switching frequency harmonics from entering the system is presented in figure 5. Here $L T$ denotes the leakage inductance of each of the Transformers, $u \cdot V d c$ denotes the switching voltage generated at the inverter output terminal. The copper loss of the Transformers and the switching losses of the inverter are modeled by the resistance $R T$. $C f$ and $L f$ represent the filter capacity and inductance.

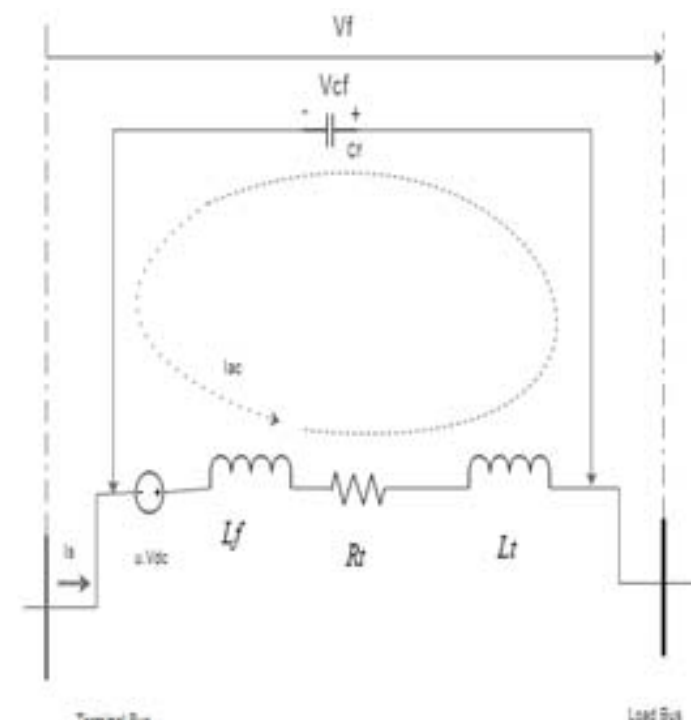

Figure 5: Single phase equivalent circuit of DVR with capacitor filter placed on the converter side of the transformer

In order to construct the state space model of the system from equivalent circuit, we define a state vector as $x T=$ [ $\left.V_{f} i_{a c}\right]$, where $V_{f}$ is the voltage at the capacitor terminals and $i_{a c}$ is the DVR current.

Using Kirchhoff's laws for the equivalent circuit from the figure 5, after some mathematical manipulations, the state space model is expressed by the following equations system:

$$
\overline{\dot{x}}=A \cdot \bar{x}+B \cdot \bar{u}
$$

$$
y=C \cdot \bar{x}+D \cdot \bar{u} \text {. }
$$

Where:

$$
\begin{gathered}
A=\left[\begin{array}{cc}
0 & \frac{1}{C_{f}} \\
-\frac{1}{L_{T}} & -\frac{R_{T}}{L_{T}}
\end{array}\right] \boldsymbol{B}=\left[\begin{array}{cc}
0 & \frac{1}{C_{f}} \\
\frac{V_{d c}}{L_{T}} & 0
\end{array}\right] \ldots \\
C=\left[\begin{array}{ll}
1 & 0
\end{array}\right] D=\left[\begin{array}{ll}
1 & 0
\end{array}\right] \quad \mathrm{y}=V_{D V R} \ldots
\end{gathered}
$$

voltage across the DC capacitor bank.

The control vector is $u T=\left[\begin{array}{ll}u c & i_{S}\end{array}\right]$, where the $u c$ is the control variable and $i_{S}$ the current that flows through the Power system.

The transfer function is determinate from the state space representation $\{\mathrm{A}, \mathrm{B}, \mathrm{C}, \mathrm{D}\}$ by applying the Laplace transform to the equation system described in (6), assuming null initial conditions. Then, the following equations system is obtained:

$$
\begin{aligned}
s \cdot \bar{X}(s)= & A \cdot \bar{X}(s)+B \cdot \bar{U}(s) \\
& \bar{Y}(s)=C \cdot \bar{X}(s)+D \cdot \bar{U}(s)
\end{aligned}
$$

From the first equations of system (9) we obtain the

$\bar{X}(s)$ Term as:

$$
\bar{X}(s)=(s \cdot I-A)^{-1} \cdot B \cdot \bar{U}(s)
$$

Substituting (10) in second equation of (9), we obtain:

$$
\bar{Y}(s)=\left(C \cdot(s . I-A)^{-1} \cdot B+D\right) \cdot \bar{U}(s) \ldots
$$

The transfer matrix function of the system is:

$$
\bar{G}(s)=\left(C .(s . I-A)^{-1} \cdot B+D\right) \ldots
$$

\section{The Dynamic Voltage Restorer Control}

The control plan for DVR compensator actualized in Matlab/Simulink is exhibited in figure 6 . The control system is intended to guarantee a symmetrical burden voltage vector at the yield terminals of controller. Under the suspicion that the framework has no zero succession segments, all streams and voltages can be remarkably changed into the Synchronous-Rotating dq reference outline.

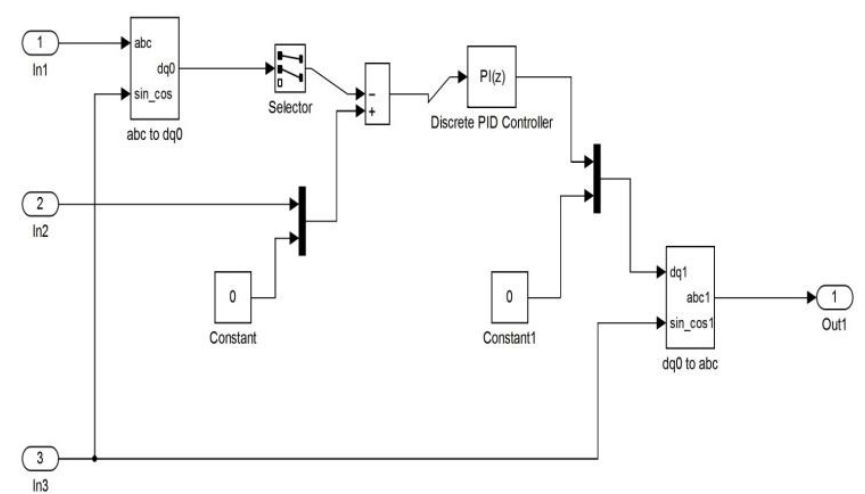

Figure 6: The voltage regulator control scheme implemented in Matlab/Simulink

Thus, the new coordinate system is defined with the $d$ - axis always coincident with the instantaneous voltage vector $(v d=|v|, \quad v q=o)$. Consequently, the $d$-axis component contributes to the instantaneous active power and $q$ - axis component contributes to the instantaneous reactive power. This operation permits to design a simpler control system than using $a b c$ component, by using a PI controller. Thus according to this theory, the load voltage vector is transformed into the $d q$ reference frame.

$V_{D V R}$ Is the series voltage injected by DVR. $V D C$ is the 


\section{International Journal of Science and Research (IJSR) \\ ISSN (Online): 2319-7064}

Index Copernicus Value (2015): 78.96 | Impact Factor (2015): 6.391

The transformation of the tree-phase instantaneous voltage frame into the synchronous-rotating $d q$ reference frame uses Park's transformation as follows:

$$
\left[\bar{u}_{d q 0}\right]=\left[T_{d q 0}\right] \cdot\left[\bar{u}_{a b c}\right]
$$

Where the Park's transformation matrix [Tdq0] is defined as

$$
\left[T_{d q 0}\right]=\frac{2}{2}\left[\begin{array}{ccc}
\cos \theta & \cos \left(\theta-\frac{2 \pi}{3}\right) & \cos \left(\theta+\frac{2 \pi}{3}\right) \\
-\sin \theta & -\sin \left(\theta-\frac{2 \pi}{3}\right) & -\sin \left(\theta+\frac{2 \pi}{3}\right) \\
\frac{1}{2} & \frac{1}{2} & \frac{1}{2}
\end{array}\right]
$$

In equation we note with $\theta$ the transformation angle calculated as in with relation:

$$
\theta(t)=\theta(0)+\int_{0}^{t} \omega(t) d t
$$

And with $\omega$ is the angular speed of the $d q$ reference frame.

The control methodology is intended to guarantee a symmetric load voltage vector and a unitary Power component to the yield terminals of controller. Therefore the $q$ component of the reference voltage is chosen zero and the $d$ component of the reference voltage is 1 .

The $d q$-components of the load voltage are compared with the reference voltages and the PI controller computes the errors, thus obtaining $d q$-components of the inverter. The inverter voltage is transformed into the $a b c$ frame.

The voltage infused by DVR is synchronized with the framework voltage utilizing a Phase Locked Loop (PLL) module. Coordinate changes from abc to dq segments in the voltage estimation framework are likewise synchronized through the PLL. The sign acquired from voltage controller is balanced utilizing Pulse Width Modulation (PWM) procedure. One of the best choices for DVR converter is viewed as the PWM, since lessens the sounds substance and prompts a quick reaction. The PWM Generator offers the terminating beats for the inverter IGBTs.

\section{Test Systems}

The Test Distribution Power System used to approve the proposed model and control system is executed in Matlab/Simulink . A 40 MVA 110/20 kV power Substation is supplied through a $10 \mathrm{~km}$ high Voltage line from the high Voltage source. A DVR is powered by a series connected batteries. A fuel system is used to charge the batteries. the system is utilized to demonstrate the capacities of the DVR compensator to repay Voltage Sags when a Fault happens in the Distribution system. The Inverter output side is connected to main supply. The compensator incorporates 3 single-stages $20 / 10 \mathrm{kV}$ Transformers associated in star design on the Inverter output side. A first three phase to ground Fault happens on the high Voltage system at time $\mathrm{t}=$ $0,2 \mathrm{~s}$. This Fault is evacuated by the System insurances after $0.15 \mathrm{~s}$. A second three phase line-line Fault happens in the medium Voltage system, at time $\mathrm{t}=0.6 \mathrm{~s}$, on another medium Voltage link supplied from the medium Voltage Bus Bar of Transformer station. This three phase Fault is evacuated by the insurances System of the high Voltage/medium Voltage Substation after $0.4 \mathrm{~s}$. These Faults that show up on nearby lines lead to Voltage plunges felt by purchasers encouraged through sound Power lines. The effect of utilizing the DVR recreation results are shown in fig 7.

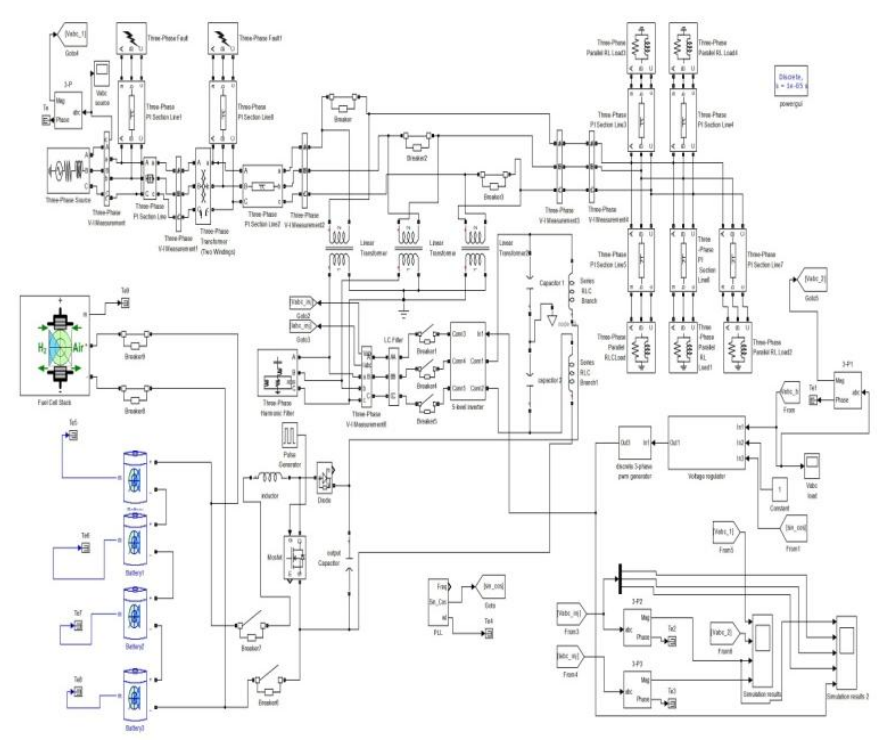

Figure 7: The Matlab/Simulink system test implementation

\section{Simulation Results and Comparision}

The first simulation was finished with no DVR. A three phase to ground fault is applied to the System at point with fault resistance of $85 \Omega$ for time duration of 0.15 seconds, also a three phase fault is applied to the system with fault resistance of $4.5 \Omega$ for time duration of 0.4 seconds. The second simulation is done at the same situation as above however a DVR is presently acquainted at the load side to compensate the voltage sag happened because of the three phase fault connected. Figure 8 shows the rms voltage at load point when the system operates without DVR and a three phase fault applied to the system. When the DVR is in operation the voltage interruption is solved almost completely and the rms voltage at the sensitive load point is maintained at normal condition.

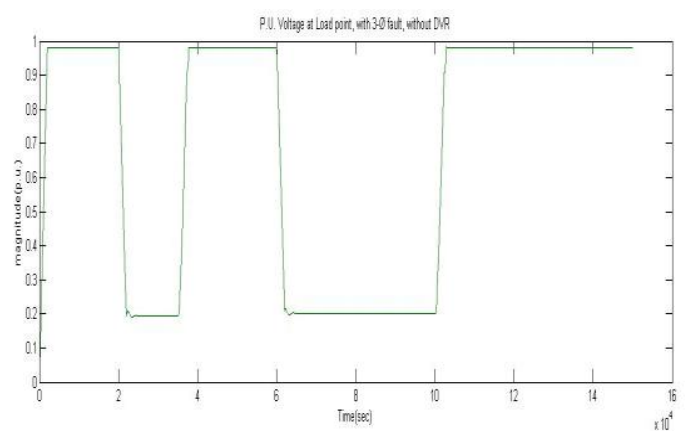

Figure 8: P.U Voltage at Load point, with $3-\varnothing$ fault, without DVR 
International Journal of Science and Research (IJSR)

ISSN (Online): 2319-7064

Index Copernicus Value (2015): 78.96 | Impact Factor (2015): 6.391

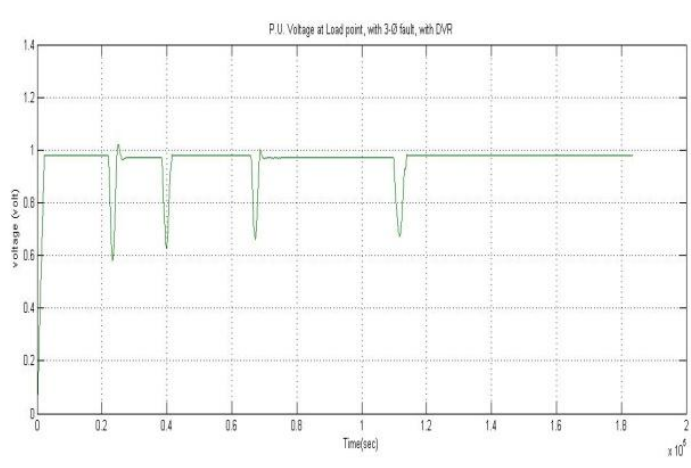

Figure 9: P.U. Voltage at Load point, with 3-Ø fault, with DVR

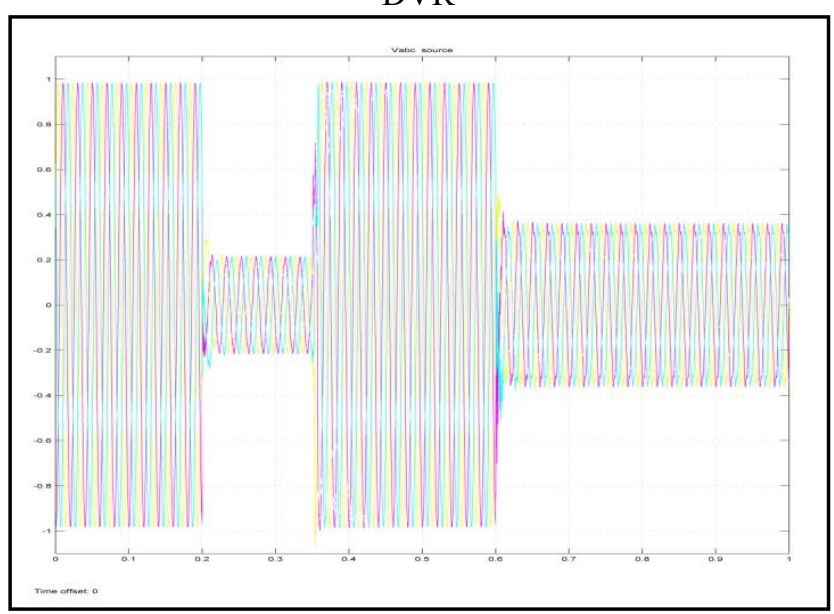

Figure 10: 3-Ø Voltage at Load point, with 3-Ø fault, without DVR

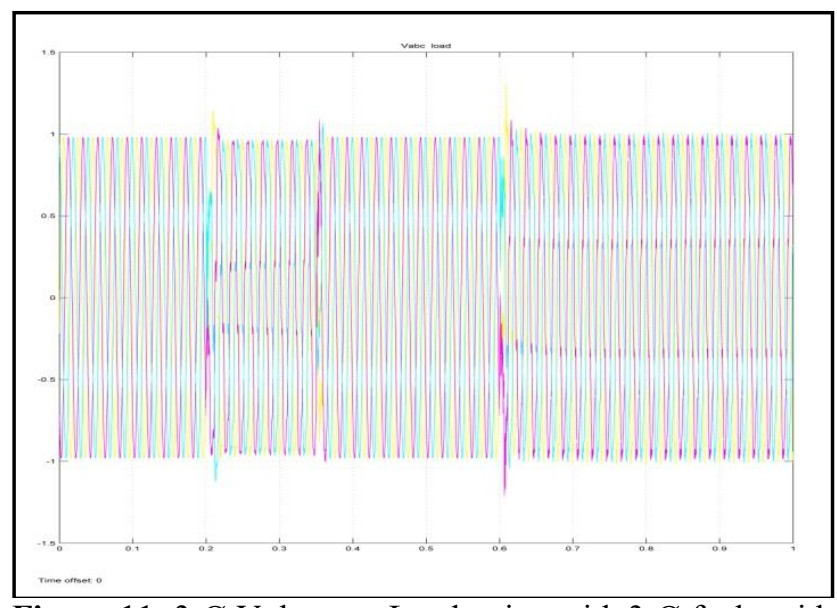

Figure 11: 3-Ø Voltage at Load point, with 3-Ø fault, with DVR

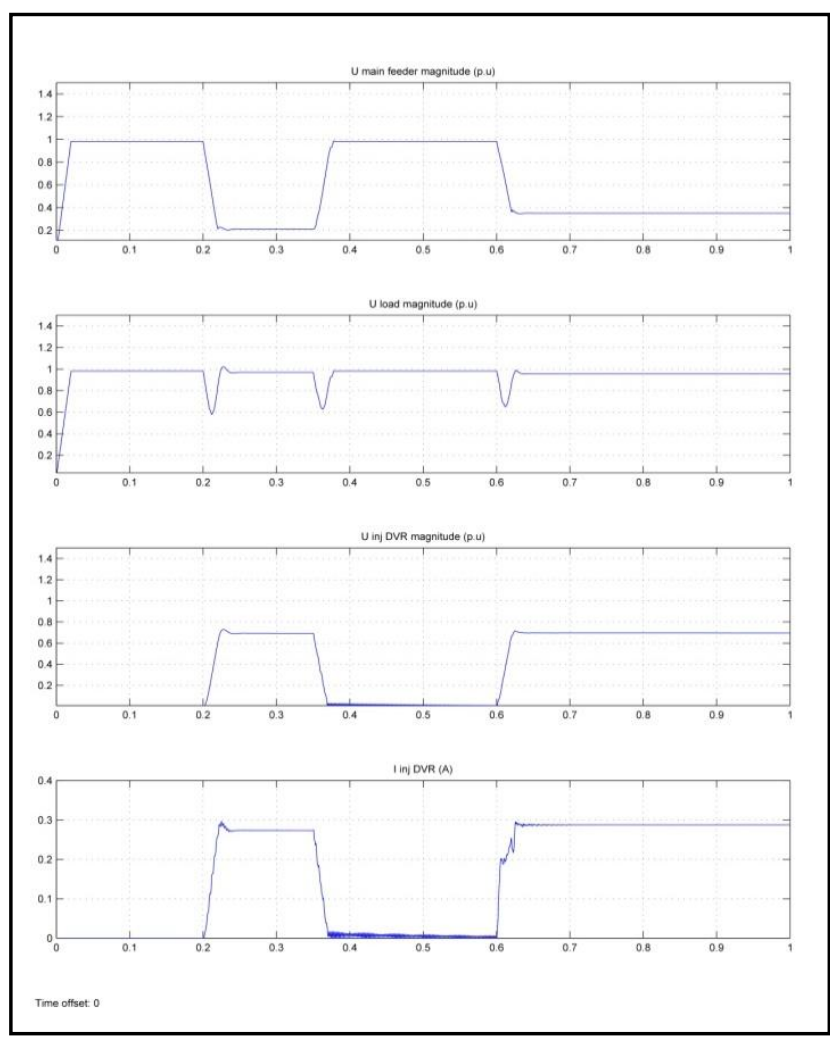

Figure 12: Simulation results: U main feeder magnitude (p.u), U load Magnitude (p.u), U inj DVR magnitude (p.u), I inj DVR (A)

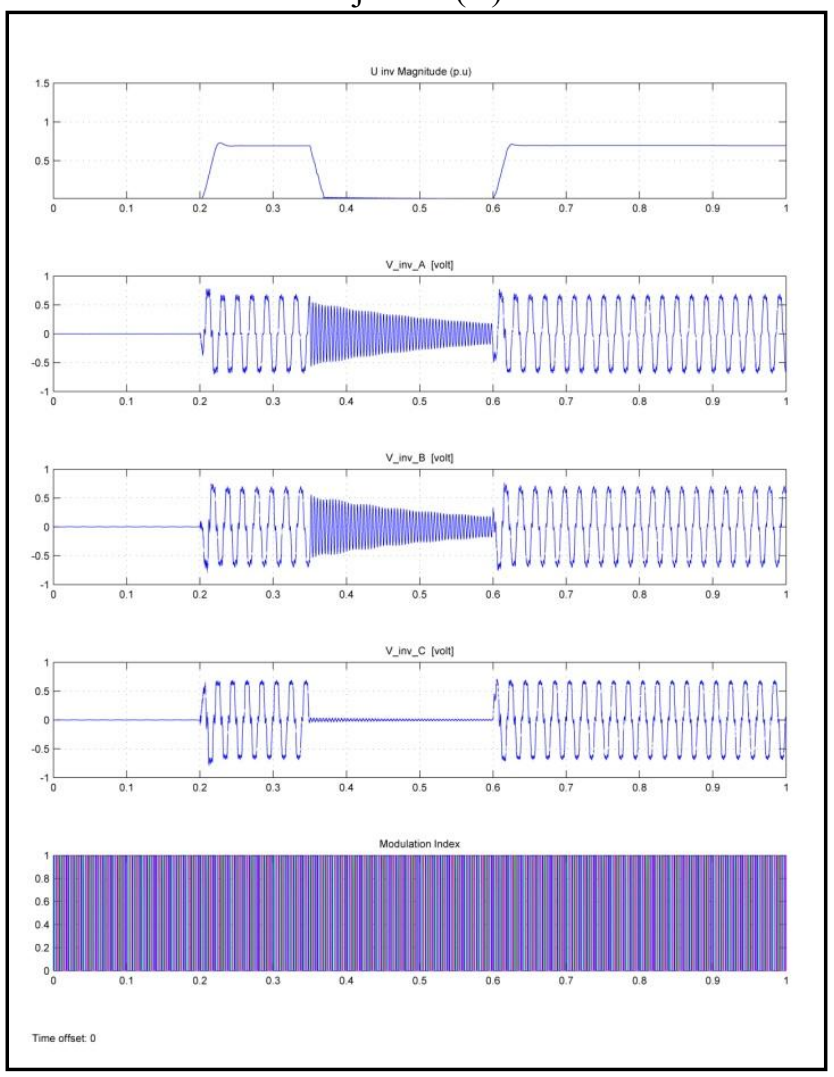

Figure 13: Simulation results: the injected inverter voltage magnitude in p.u., the inverter voltages curves and firing pulses

\section{Conclusion}

This thesis advise a technique to compensate voltage dips in medium voltage networks. The compensation is performed 


\section{International Journal of Science and Research (IJSR) \\ ISSN (Online): 2319-7064}

Index Copernicus Value (2015): 78.96 | Impact Factor (2015): 6.391

by using a dynamic voltage restorer (DVR)). The chosen configuration of dynamic voltage restorer and its control strategy for voltage level restoration on the load bus bar of a medium voltage connection substation are also presented. The electrical distribution network and the dynamic voltage restorer are modeled using Matlab / Simulink simulation environment in order to demonstrate the effectiveness of proposed equipment. The simulations completed demonstrated that the picked setup of DVR gives incredible voltage compensation abilities.

\section{Refrences}

[1] Rai, A.K. Nadir - "Modeling \& Simulation of Dynamic Voltage Restorer (DVR) for Enhancing Voltage Sag", Sensors \& Transducers Journal, Vol. 87, Issue 1, pp. 85933, January 2008

[2] M.H.J. Bollen - „Understanding ppower quality problems, voltage sags and interruptions”, IEEE Press, New York, 2000,

[3] Ghosh, G. Ledwich - "Power Quality Enhancement using Custom Power Devices", Kluwer Academiic Publishers, Boston/Dordrecht/Lon- don , 2002

[4] Gheorghe-Ioan Nicolaescu, Horia Andrei, Stefan Radulescu "Modeling and Simulation of Dynamic Voltage Restorer for Voltage Sags Mitigation in Medium Voltage Networks with Secondary Distribution Configuration' Electrical Engineering Dept., University Valahia, Targoviste, Romania

[5] J.G. Nielsen - "Design and Control of a Dynamic Voltage Restorer", Aalborg University, ISBN: 8789179-42-0, Denmark, 2002

[6] S. Paul, S. Sarkar, P.K. Saha, G.K. Panda - "Dynamic Voltage Restores for power quality Improvement", International Journal Of Engineering And Computer Science, ISSN:2319-7242, Volume 2, Issue 1, Page No. 234-239, Jan 2013

[7] R. Omar, N.A.Rahim, M. Sulaiman - „Dynamic Voltage Restorer for Power Quality Improvement in Electrical Distribution System: An Overview", Australian Journal of Basic and Applied Sciences, vol. 5 (12), pp. 379-396, 2001

[8] M.H. Haque - "Compensation of distribution system voltage sag by DVR and D-STATCOM", IEEE Porto Power Tech Conference, 2001

[9] J.G. Nielsen, F. Blaabjerg - „Comparison of System topologies for Dynamic Voltage Restorer", IAS 2001, Chicago

[10] J.G. Nielsen - „Analysis of topologies for Dynamic Voltage Restorer", Nor-DAC 2000, Trondheim 